Pacific Journal of Mathematics

AN ERROR ESTIMATE UNIFORM IN TIME FOR SPECTRAL
GALERKIN APPROXIMATIONS OF THE NAVIER-STOKES
PROBLEM

JohN GRoves HEYwood 


\title{
AN ERROR ESTIMATE UNIFORM IN TIME FOR SPECTRAL GALERKIN APPROXIMATIONS OF THE NAVIER-STOKES PROBLEM
}

\author{
JoHN G. HEYWOOD
}

1. Introduction. The existence theory for the nonstationary Navier-Stokes equations can be developed, by the method of Galerkin approximation, using any of a wide variety of possible systems of basis functions. The basis functions used in the papers of Hopf [8] and of Kiselev and Ladyzhenskaya [10] are merely assumed to belong to and be complete in certain function spaces. However, to obtain refinements in the theory by the Galerkin method, particularly regarding the regularity and decay of solutions, it often appears essential to choose as basis functions the eigenfunctions of the Stokes operator; see Ito [9], Lions [12], Prodi [14], Foias [3], Ladyzhenskaya [11], Temam [17], and Heywood [4, 5, 6].

In these and other works, the convergence of the Galerkin approximations is generally proved by a compactness argument, based on $a$-priori bounds for the approximations. A notable exception is the paper [3] of Foias, where (on page 324) the approximations are shown to converge, uniformly over a time interval, in the Dirichlet norm. Recently, Rautmann $[15,16]$ has drawn attention to this type of result, and gone further, giving a systematic development of error estimates, for the Galerkin approximations and their time derivatives.

Rautmann's error estimates (and also Foias' convergence theorem) are presented locally, valid on a finite interval determined by certain norms of the data. At best, if one assumes the solution to be approximated is uniformly regular, for $t \in[0, \infty)$, the method yields an error estimate which grows exponentially with time. And, without further assumptions, this is the best that can be expected. However, if one assumes, additionally, the solution to be approximated is stable, then it is reasonable to expect an error estimate which is uniform in time. This is what is done in the present paper. It is hoped the result may prove suggestive for future developments in the NavierStokes theory. It should be mentioned, however, the original reason for undertaking this work arose in the author's joint study [7] with Rolf Rannacher, of finite element Galerkin approximations, a context in which error estimates uniform in time have important implications for computations. While the present work served to fix some ideas, in a simpler, purely theoretical context, it has turned out our arguments in the finite element context are substantially different. 
We present here only the most basic result of its type. However, a number of elaborations seem possible, some of which are being investigated with Rannacher in the numerical context. We mention, the stability condition (A3) is formulated here in terms of the Dirichlet norm, could be formulated in terms of the $L^{2}$-norm. Also, our error estimate is given in the Dirichlet norm; it should be possible to obtain an improved rate of convergence in the $L^{2}$-norm. Finally, in some situations, there exist "stable" solutions which do not possess all the spatial symmetry of the domain and external forces. Of course, in such situations, there is only stability modulo shifts in the spatial symmetry. The definition of stability we work with, here, is too strict to allow for this possibility. We think it is possible to weaken our definition of stability, to be neutral relative to drifts with respect to the spatial symmetry, resulting in error estimates modulo shifts in the symmetry. Similarly, if the boundary values and forces are time independent, stability and error estimates may be considered modulo drifts and shifts in time.

After a short preliminary $\S 2$, our result is stated in $\S 3$, and proved in $\S \S 4$ through 7 .

2. Preliminaries. Let $\Omega \subset R^{n}, n=2$ or 3 , be a bounded domain with smooth boundary $\partial \Omega$. The function spaces customarily denoted by $L^{P}(\Omega), H^{m}(\Omega), C_{0}^{\infty}(\Omega)$ are defined as usual, though we will not distinguish in our notation between spaces of $R$ and of $R^{n}$-valued functions, the distinction always being clear from the context. The $L^{2}$ inner-product and norm are denoted by $(\cdot, \cdot)$ and $\|\cdot\|$ respectively, and the $L^{r}$ norm by $\|\cdot\|_{p}$. The following spaces of solenoidal functions are, of course, $R^{n}$-valued:

$$
\begin{aligned}
& D(\Omega)=\left\{\dot{\phi}: \phi \in C_{0}^{\infty}(\Omega) \text { and } \nabla \cdot \phi=0\right\}, \\
& J(\Omega) \text { is the completion of } D(\Omega) \text { in } L^{2}(\Omega), \\
& J_{1}(\Omega) \text { is the completion of } D(\Omega) \text { in } H^{1}(\Omega) .
\end{aligned}
$$

We let $P$ denote the orthogonal projection $L^{2}(\Omega) \rightarrow J(\Omega)$, and let $\tilde{\Delta}=P \Delta$, where $\Delta$ is the Laplacean operator. We list, as lemmas, some results which will be needed later.

Lemma1. For $u \in H^{1}(\Omega),\|P u\|_{H^{1}} \leqq c\|u\|_{I^{1}}$.

LEMMA 2. For every $u \in J(\Omega) \cap H^{1}(\Omega)$, there exists a function is $\in H^{2}(\Omega)$ such that $\operatorname{curl} \psi=u$, $\left.\psi\right|_{\partial \Omega}=0$, and $\|\psi\|_{I I^{2}} \leqq c\|u\|_{I I^{1}}$.

LeMma 3. For $g \in L^{2}(\Omega)$, the unique solution $v \in J_{1}(\Omega), q \in L^{2}(\Omega) / R$ of the Stokes problem 


$$
-\Delta_{q}+\nabla_{v}=g \text { and } \nabla \cdot v=0 \text { in } \Omega,\left.\quad v\right|_{\partial \Omega}=0,
$$

satisfies $\|v\|_{H^{2}}+\|q\|_{I^{1 / R}} \leqq c\|g\|$. Hence, also, $\|v\|_{I^{2}} \leqq c\|\widetilde{\Delta} v\|$ holds for all $v \in J_{1}(\Omega) \cap H^{2}(\Omega)$.

For proofs of Lemmas 1 and 3 , see [17, p. 18] and [1], respectively. In Lemma 2 , if $\Omega$ is two-dimensional, it should be understood that $\psi$ is scalar valued and that $\operatorname{curl} \psi=\left(\partial \psi / \partial x_{2},-\partial \psi / \partial x_{1}\right)$. The proof in the more difficult three-dimensional case is as follows. By a well-known construction $\left[11\right.$, p. 25], $u$ can be continued to all $R^{3}$ as a solenoidal function $u \in H^{1}\left(R^{3}\right)$ with compact support. Hence it can be expressed as $u=\operatorname{curl} \phi$, in terms of the vector potential $\phi^{\prime}(x)=(4 \pi)^{-1} \int(\operatorname{curl} u) / r d y$. Since $(\operatorname{curl} \phi) \cdot n=0$ on $\partial \Omega$, a scalar function $p$ can be defined on component $\partial \Omega_{k}$ of $\partial \Omega$ by setting $p(x)=\int_{C} \phi \cdot d s$, where $C$ is any curve lying in $\partial \Omega$ joining $x$ to a fixed point of $\partial \Omega_{k}$. Since $\phi \in H^{2}(\Omega)$, we have $p \in W_{2}^{5 / 2}(\Omega \partial)$ and $\phi \cdot n \in W_{2}^{3 / 2}(\partial \Omega)$. It follows that $p$ can be continued into $\Omega$ as a function $p \in H^{3}(\Omega)$ satisfying $\partial p / \partial n=\phi \cdot n$ on $\partial \Omega$; see $[13$, p. 104]. Clearly $\nabla p=\phi$ on $\partial \Omega$, and thus $\psi=\dot{\phi}-\nabla p$ is a function with all the desired properties.

We denote by $\left\{a^{k}(x)\right\}$ and $\left\{\lambda_{k}\right\}$ the eigenfunctions and eigenvalues of the Stokes operator $\widetilde{J}$ defined in $J_{1}(\Omega) \cap H^{2}(\Omega)$, i.e., of the problem $-\Delta v+\nabla q=\lambda v$ and $\nabla \cdot v=0$ in $\Omega,\left.v\right|_{\partial \Omega}=0$. Thus $-\widetilde{\Delta} a^{k}=\lambda_{k} a^{k}$. Some well known results and elementary observations are collected in the following lemma.

Lemma 4. The eigenfunctions $\left\{a^{k}\right\}$ are orthogonal in the inner products $(u, v),(\nabla u, \nabla v)$, and $(\widetilde{\Delta} u, \widetilde{\Delta} v)$, and complete in the spaces $J(\Omega), J_{1}(\Omega)$ and $J_{1}(\Omega) \cap H^{2}(\Omega)$. If $\sum_{k=1}^{\infty} c_{k} a^{k} \in J_{1}(\Omega)$, then

$$
\left\|\sum_{k=n}^{\infty} c_{k} a^{k}\right\|^{2} \leqq \lambda_{n}^{-1}\left\|\nabla \sum_{k=n}^{\infty} c_{k} a^{k}\right\|^{2} .
$$

If $\sum_{k=1}^{\infty} c_{k} a^{k} \in J_{1}(\Omega) \cap H^{2}(\Omega)$, then

$$
\left\|\nabla \sum_{k=n}^{\infty} c_{k} a^{k}\right\|^{2} \leqq \lambda_{n}^{-1}\left\|\tilde{\Delta} \sum_{k=n}^{\infty} c_{k} a^{k}\right\|^{2}
$$

We list, in the following lemma, some Poincaré and Sobolev inequalities that will be needed, sometimes combined with the results of Lemmas 3 and 4 . We will use only the three-dimensional versions of Sobolev's inequalities, these being, of course, also valid in bounded two-dimensional domains.

LemmA 5. If $u \in J_{1}(\Omega) \cap H^{2}(\Omega)$, then $\|u\| \leqq \lambda_{1}^{-1 / 2}\|\nabla u\| \leqq \lambda_{1}^{-1}\|\widetilde{\Delta} u\|$, 


$$
\|u\|_{6} \leqq c\|\nabla u\|, \quad \sup |u| \leqq c\|\widetilde{\Delta} u\|, \quad\|u\|_{+} \leqq c\|u\|^{1 / 4}\|\nabla u\|^{3 / 4},
$$

and $\|\nabla u\|_{6} \leqq c\|\widetilde{\Delta} u\|$.

3. Statement of the result. Let $u(x, t), p(x, t)$ be a solution of the Navier-Stokes problem:

$$
\begin{aligned}
& u_{t}-\Delta u+u \cdot \Delta u+\nabla p=f \text { and } \\
& \nabla \cdot u=0 \text { in } \quad \Omega \times(0, \infty), \\
& \left.u\right|_{t=0}=a,\left.\quad u\right|_{\partial \Omega}=0 .
\end{aligned}
$$

We assume the data for problem (1) satisfies

$$
a \in J_{1}(\Omega) \cap H^{2}(\Omega), \quad \sup _{t \geqq 0}\|f\|<\infty, \quad \sup _{t \geqq 0}\left\|f_{t}\right\|<\infty,
$$

and that the solution satisfies

$$
\|\nabla u(t)\| \leqq M, \quad \text { for all } t \geqq 0 .
$$

We further assume $u$ is conditionally exponentially stable, in the sense of condition (A3), below. The stability condition concerns the behaviour of perturbations of $u$. A function $\zeta(x, t)$, defined on some interval $t \geqq t_{0}$, is called a perturbation of $u$, if $\zeta+u$ is a solution of the Navier-Stokes equations, and if $\zeta_{\partial \Omega}=0$. Thus, setting $\zeta_{0} \equiv$ $\zeta\left(\cdot, t_{0}\right), \zeta$ is a solution of the initial-boundary value problem:

$$
\begin{aligned}
& \zeta t-\Delta \zeta+u \cdot \nabla \zeta+\zeta \cdot \nabla u+\zeta \cdot \nabla \zeta+\nabla q=0 \text { and } \\
& \nabla \cdot \zeta=0 \text { in } \Omega \times\left(t_{0}, \infty\right), \\
& \left.\zeta\right|_{t=t_{0}}=\zeta_{0},\left.\quad \zeta\right|_{\partial \Omega}=0 .
\end{aligned}
$$

Our assumption, then, is:

(A3) There exist positive numbers $\alpha, A, \delta$ such that for every $t_{0} \geqq 0$, and every $\zeta_{0} \in J_{1}(\Omega) \cap H^{2}(\Omega)$ with $\left\|\nabla \zeta_{0}\right\|<\delta$, the perturbation problem (2) is uniquely solvable and its solution satisfies $\|\nabla \zeta(t)\| \leqq$ $A\left\|\nabla \zeta_{0}\right\| e^{-\alpha\left(t-t_{0}\right)}$, for all $t \geqq t_{0}$.

The $n$th spectral Galerkin approximation

$$
u^{n}(x, t)=\sum_{k=1}^{n} c_{k n}(t) a^{k}(x)
$$

to the solution of problem (1) is uniquely determined by the conditions

$$
\begin{aligned}
& \left(u_{t}^{n}, \phi^{n}\right)+\left(\nabla u^{n}, \nabla \phi^{n}\right)+\left(u^{n} \cdot \nabla u^{n}, \phi^{n}\right)=\left(f, \phi^{n}\right), \text { for } t \geqq 0, \text { and } \\
& \left(u^{n}(0)-a, \phi^{n}\right)=0,
\end{aligned}
$$

for all $\phi^{n}$ of the form $\phi^{n}(x)=\sum_{k=1}^{n} d_{k} a^{k}(x)$.

TheOREM. There exist constants $N$ and $K$ depending only on 
the domain $\Omega$, the norms of the data referred to in (A1), and the constants intrduced in (A2) and (A3), such that

$$
\left\|\nabla\left(u-u^{n}\right)(t)\right\| \leqq K \lambda_{n+1}^{-1 / 2},
$$

for all $t \geqq 0$, provided $n \geqq N$.

4. Comparison of error with perturbations. Let $u=$ $\sum_{k=1}^{\infty} c_{k}(t) a^{k}(x)$ be the eigenfunction expansion of the solution $u$ of problem (1). Let $v^{n}=\sum_{k=1}^{n} c_{k}(t) a^{k}(x)$ be the $n$th partial sum of the series for $u$. Let $e^{n}=u-v^{n}$, and let $\eta^{n}=u^{n}-v^{n}$, were $u^{n}$ is the $n$th Galerkin approximation. Then $u-u^{n}=e^{n}-\eta^{n}$.

Lemma 6. The assumptions (A1), (A2) imply $\|\widetilde{\Delta} u(t)\|,\left\|u_{t}(t)\right\|$, and $e^{-t} \int_{0}^{t} e^{\tau}\left\|\nabla u_{t}\right\|^{2} d \tau$ are uniformly bounded, for $t \geqq 0$. Thus, $\left\|\nabla e^{n}(t)\right\| \leqq c \lambda_{n+1}^{-1 / 2}$ and $\left\|e^{n}(t)\right\| \leqq c \lambda_{n+1}^{-1}$, for $t \geqq 0$.

These $a$-priori estimates for $u$ are proved in [7]; most of the argument is repeated, in a slightly different context, in Lemma 8 below. The estimates for $e^{n}$ follow, using Lemma 4 .

It remains to estimate $\eta^{n}$. Observe that $v^{n}$ satisfies the linearized equations

$$
\left(v_{t}^{n}, \phi^{n}\right)+\left(\nabla v^{n}, \nabla \phi^{n}\right)+\left(u \cdot \nabla u, \phi^{n}\right)=\left(f, \phi^{n}\right), \quad \text { for } t \geqq 0,
$$

for all $\phi^{n}$ of the form $\phi^{n}(x)=\sum_{k=1}^{n} d_{k} a^{k}(x)$. This is easily seen starting with the weak Navier-Stokes equations for $u$ and using the orthogonality relations for the $\left\{a^{k}\right\}$. Subtracting (5) from (3) gives

$$
\begin{aligned}
\left(\eta_{t}^{n}, \phi^{n}\right)+\left(\nabla \eta^{n}, \nabla \phi^{n}\right)= & \left(u \cdot \nabla u, \phi^{n}\right)-\left(u^{n} \cdot \nabla u^{n}, \phi^{n}\right) \\
= & -\left(u \cdot \nabla \eta^{n}, \phi^{n}\right)-\left(\eta^{n} \cdot \nabla u^{n}, \phi^{n}\right) \\
& +\left(u \cdot \nabla e^{n}, \phi^{n}\right)+\left(e^{n} \cdot \nabla u^{n}, \phi^{n}\right) \\
= & -\left(u \cdot \nabla \eta^{n}, \phi^{n}\right)-\left(\eta^{n} \cdot \nabla u, \phi^{n}\right)-\left(\eta^{n} \cdot \nabla \eta^{n}, \phi^{n}\right) \\
& +\left(\eta^{n} \cdot \nabla e^{n}, \phi^{n}\right)+\left(e^{n} \cdot \nabla \eta^{n}, \phi^{n}\right) \\
& +\left(u \cdot \nabla e^{n}, \phi^{n}\right)+\left(e^{n} \cdot \nabla v^{n}, \phi^{n}\right) .
\end{aligned}
$$

This identity will be used, in $\S 5$, to get an $a$-priori estimate for $\left\|\widetilde{\Delta \eta^{n}}(t)\right\|$. In order to compare $\eta^{n}$ with a perturbation $\zeta$, we must rewrite (6) in a form valid for all test functions $\phi(x) \in J_{1}(\Omega) \cap H^{2}(\Omega)$.

Let $P_{n}$ and $Q_{n}$ be the orthogonal projections of $L^{2}(\Omega)$ onto $\operatorname{Span}\left\{a_{1}, \cdots, a_{n}\right\}$ and $\operatorname{Span}\left\{a_{n+1}, a_{n+2}, \cdots\right\}$, respectively. Clearly $P$, $P_{n}$ and $Q_{n}$ all commute, $P=P_{n}+Q_{n}$, etc. For $\phi \in J(\Omega)$, let us write $\phi=P_{n} \phi+Q_{n} \phi=\phi^{n}+Q_{n} \phi$. Then, for any $w \in L^{2}(\Omega)$,

$$
\left(w, \phi^{n}\right)=\left(P_{n} w, \phi\right)=(w, \phi)-\left(Q_{n} w, \phi\right) .
$$


Also, for $\phi \in J_{1}(\Omega)$,

$$
\left(\nabla \eta^{n}, \nabla \phi^{n}\right)=-\left(\widetilde{\Delta} \eta^{n}, \dot{\phi}^{n}\right)=-\left(\widetilde{\Delta} \eta^{n}, \phi\right)=\left(\nabla \eta^{n}, \nabla \phi\right) .
$$

Using such obvious identities, we rewrite (6) as

$$
\begin{aligned}
\left(\eta_{t}^{n}, \phi\right)+ & \left(\nabla \eta^{n}, \nabla \dot{\phi}\right)+\left(u \cdot \nabla \eta^{n}, \phi\right)+\left(\eta^{n} \cdot \nabla u, \phi\right)+\left(\eta^{n} \cdot \nabla \eta^{n}, \phi\right) \\
= & \left(Q_{n}\left[u \cdot \nabla \eta^{n}\right], \phi\right)+\left(Q_{n}\left[\eta^{n} \cdot \nabla u\right], \phi\right)+\left(Q_{n}\left[\eta^{n} \cdot \nabla \eta^{n}\right], \phi\right) \\
& +\left(P_{n}\left[\eta^{n} \cdot \nabla e^{n}\right], \phi\right)+\left(P_{n}\left[e^{n} \cdot \nabla \eta^{n}\right], \phi\right) \\
& +\left(P_{n}\left[u \cdot \nabla e^{n}\right], \phi\right)+\left(P_{n}\left[e^{n} \cdot \nabla v^{n}\right], \phi\right),
\end{aligned}
$$

which is valid for all $\phi \in J(\Omega)$, and $t \geqq 0$. This is to be compared with the weak form of (2), which is

$$
\left(\zeta_{t}, \phi\right)+(\nabla \zeta, \nabla \phi)+(u \cdot \nabla \zeta, \phi)+(\zeta \cdot \nabla u, \phi)+(\zeta \cdot \nabla \zeta, \phi)=0 .
$$

Let us denote the right side of $(7)$ by $\left(g^{n}, \phi\right)$, and let $w=\eta^{n}-\zeta$. Then, subtracting (8) from (7) gives

$$
\begin{gathered}
\left(w_{t}, \phi\right)+(\nabla w, \nabla \phi)+(u \cdot \nabla w, \phi)+(w \cdot \nabla u, \phi) \\
+\left(\eta^{n} \cdot \nabla w, \phi\right)+(w \cdot \nabla \zeta, \phi)=\left(g^{n}, \phi\right),
\end{gathered}
$$

for all $\phi \in J(\Omega)$, and $t \geqq t_{0}$. The regularity of $\eta^{n}$ and $\zeta$, which is implicitely assumed here and in Lemma 7 below, will be verified later. Setting $\phi=-\widetilde{\Delta} w$, we obtain

$$
\begin{aligned}
\frac{1}{2} \frac{d}{d t}\|\nabla w\|^{2}+\|\tilde{\Delta} w\|^{2}= & (u \cdot \nabla w, \widetilde{\Delta} w)+(w \cdot \nabla u, \widetilde{\Delta} w) \\
& +\left(\eta^{n} \cdot \nabla w, \widetilde{\Delta} w\right)+(w \cdot \nabla \zeta, \widetilde{\Delta} w)-\left(g^{n}, \widetilde{\Delta} w\right) \\
\leqq & c\|\widetilde{\Delta} u\| \cdot\|\nabla w\| \cdot\|\widetilde{\Delta} w\|+c\left\|\widetilde{\Delta} \eta^{n}\right\| \cdot\|\nabla w\| \cdot\|\widetilde{\Delta} w\| \\
& +c\|\widetilde{\Delta} \zeta\| \cdot\|\nabla w\| \cdot\|\widetilde{\Delta} w\|+\left\|g^{n}\right\| \cdot\|\widetilde{\Delta} w\|,
\end{aligned}
$$

or

$$
\frac{d}{d t}\|\nabla w\|^{2}+\|\tilde{\Delta} w\|^{2} \leqq c\left(\|\widetilde{\Delta} u\|^{2}+\left\|\widetilde{\Delta} \eta^{n}\right\|^{2}+\|\tilde{\Delta} \zeta\|^{2}\right)\|\nabla w\|^{2}+\|g\|^{2},
$$

and hence:

LemMa 7. Let $t_{0} \geqq 0$ and $\zeta$ be as in problem (2). Then, for $w$ and $g^{n}$ as above, and $\beta(t)=c\left(\|\widetilde{\Delta} u\|^{2}+\left\|\widetilde{\Delta} \eta^{n}\right\|^{2}+\|\widetilde{\Delta} \zeta\|^{2}\right)$, there holds

$$
\|\nabla w(t)\|^{2} \leqq e^{\int_{t_{0} \beta(\tau) d \tau}}\left\{\left\|\nabla w\left(t_{0}\right)\right\|^{2}+\int_{t_{0}}^{t}\left\|g^{n}\right\|^{2} d \tau\right\},
$$

for all $t \geqq t_{0}$.

REMARK. It is well to point out, here, why we don't assume $\|\zeta(t)\| \leqq A\left\|\zeta\left(t_{0}\right)\right\| e^{-\alpha\left(t-t_{0}\right)}$ in (A3), in place of the condition for the 
Dirichlet norm, and then try to use a continuous dependence theorem in the $L^{2}$ norm, in place of (11). The difficulty is that we would still need to estimate $\left\|g^{n}\right\|$ and hence $\left\|\widetilde{\Delta \eta^{n}}\right\|$, and this does not seem possible, in three-dimensions, starting with an estimate for $\|w(t)\|$. But we can, starting with an estimate for $\|\nabla w(t)\|$, because this implies an estimate for $\left\|\nabla \eta^{n}\right\|$, which leads to an estimate for $\left\|\widetilde{\Delta \eta^{n}}\right\|$.

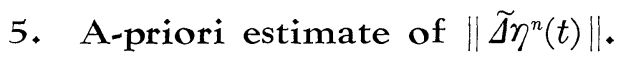

LEMma 8. Suppose $\left\|\nabla \eta^{n}(t)\right\|<\gamma$ holds on some interval $0 \leqq t \leqq t^{*}$, for some number $\gamma \leqq \delta$. Then there also holds $\left\|\widetilde{\Delta} \eta^{n}(t)\right\|^{2}<R\left(\gamma^{2}+\lambda_{n+1}^{-1}\right)$, for $0 \leqq t \leqq t^{*}$, with a constant $R$ dependent only on $\Omega, \delta$, $\sup _{t \geqq 0}\|\widetilde{\Delta} u(t)\|, \sup _{t \geqq 0}\left\|u_{t}(t)\right\|$, and $\sup _{t \geqq 0} e^{-t} \int_{0}^{t} e \tau\left\|\nabla u_{t}\right\|^{2} d \tau$, i.e., quantities all bounded via Lemma 6 in terms of the assumptions (A1), (A2), (A3).

Proof. What follows is based on the identity (6). Since the Galerkin approximation $u^{n}$ does not appear, there will be no ambiguity in setting $\eta=\eta^{n}, e=e^{n}, v=v^{n}, \phi=\phi^{n}$. Setting $\phi=-\widetilde{\Delta \eta}$ in (6), we obtain

$$
\begin{aligned}
\frac{1}{2} \frac{d}{d t}\|\nabla \eta\|^{2} & +\|\widetilde{\Delta} \eta\|^{2} \leqq c\|\widetilde{\Delta} u\| \cdot\|\nabla \eta\| \cdot\|\widetilde{\Delta \eta}\|+c\|\nabla \eta\|^{3 / 2}\|\widetilde{\Delta \eta}\|^{3 / 2} \\
& +c\|\widetilde{\Delta} u\| \cdot \lambda_{n+1}^{-1 / 2}\|\widetilde{\Delta} u\| \cdot\|\widetilde{\Delta} \eta\|,
\end{aligned}
$$

and hence

$$
\frac{d}{d t}\|\Delta \eta\|^{2}+\|\widetilde{\Delta} \eta\|^{2} \leqq c\|\widetilde{\Delta} u\|^{2} \cdot\|\nabla \eta\|^{2}+c\|\nabla \eta\|^{6}+c \lambda_{n+1}^{-1}\|\widetilde{\Delta} u\|^{4} .
$$

Multiplying (12) by $e^{t}$ and integrating gives

$$
\begin{aligned}
e^{-t} \int_{0}^{t} e^{\tau}\|\widetilde{\Delta} \eta\|^{2} d \tau & \leqq e^{-t} \int_{0}^{t} e^{\tau}\left\{\gamma^{2}+c\|\widetilde{\Delta} u\|^{2} \gamma^{2}+c \gamma^{6}+c \lambda_{n+}^{-1}\|\widetilde{\Delta} u\|^{4}\right\} d \tau \\
& \leqq e^{-t} \int_{0}^{t} e^{\tau} c\left(\gamma^{2}+\lambda_{n+1}^{-1}\right) d \tau .
\end{aligned}
$$

Setting $\phi=\eta_{t}$ in (6), we obtain

$$
\begin{aligned}
\left\|\eta_{t}\right\|^{2} \leqq & \|\widetilde{\Delta} \eta\| \cdot\left\|\eta_{t}\right\|+c\|\tilde{\Delta} u\| \cdot\|\nabla \eta\| \cdot\left\|\eta_{t}\right\|+c\|\widetilde{\Delta \eta}\| \cdot\|\nabla \eta\| \cdot\left\|\eta_{t}\right\| \\
& +c\|\tilde{\Delta} e\| \cdot\|\nabla \eta\| \cdot\left\|\eta_{t}\right\|+c\|\widetilde{\Delta} u\| \cdot\|\nabla e\| \cdot\left\|\eta_{t}\right\|,
\end{aligned}
$$

and hence

$$
\left\|\eta_{t}\right\|^{2} \leqq c\|\widetilde{\Delta} \eta\|^{2}+c\|\tilde{\Delta} u\|^{2} \cdot\|\nabla \eta\|^{2}+c\|\widetilde{\Delta} \eta\|^{2} \cdot\|\nabla \eta\|^{2}+c \lambda_{n+1}^{-1}\|\widetilde{\Delta} u\|^{+} .
$$

Multiplying by $e^{t}$ and integrating, and using (13), we get 


$$
e^{-t} \int_{0}^{t} e^{\tau}\left\|\eta_{t}\right\|^{2} d \tau \leqq e^{-t} \int_{0}^{t} e^{\tau} c\left(\gamma^{2}+\lambda_{n+1}^{-1}\right) d \tau
$$

Now differentiating (6) with respect to $t$ and setting $\phi=\eta_{t}$, we obtain

$$
\begin{aligned}
\frac{1}{2} \frac{d}{d t}\left\|\eta_{t}\right\|^{2}+\left\|\nabla \eta_{t}\right\|^{2}= & -\left(u_{t} \cdot \nabla \eta, \eta_{t}\right)-\left(\eta_{t} \cdot \nabla u, \eta_{t}\right)-\left(\eta \cdot \nabla u_{t}, \eta_{t}\right) \\
& -\left(\eta_{t} \cdot \nabla \eta, \eta_{t}\right)+\left(\eta_{t} \cdot \nabla e, \eta_{t}\right)+\left(\eta \cdot \nabla e_{t}, \eta_{t}\right) \\
& +\left(e_{t} \cdot \nabla \eta, \eta_{t}\right)+\left(u_{t} \cdot \nabla e, \eta_{t}\right)+\left(u \cdot \nabla e_{t}, \eta_{t}\right) \\
& +\left(e_{t} \cdot \nabla v, \eta_{t}\right)+\left(e \cdot \nabla v_{t}, \eta_{t}\right) \\
\leqq & c\left\|u_{t}\right\| \cdot\|\widetilde{\Delta} \eta\| \cdot\left\|\nabla \eta_{t}\right\|+c\|\widetilde{\Delta}\| \cdot\left\|\eta_{t}\right\| \cdot\left\|\nabla \eta_{t}\right\| \\
& +c\|\nabla \eta\| \cdot\left\|\eta_{t}\right\|^{1 / 2}\left\|\nabla \eta_{t}\right\|^{3 / 2}+\left\|u_{t}\right\|_{3} \cdot\|e\|_{6} \cdot\left\|\nabla \eta_{t}\right\| \\
& +c\|\widetilde{\Delta} u\| \cdot\left\|e_{t}\right\| \cdot\left\|\nabla \eta_{t}\right\|,
\end{aligned}
$$

and hence

$$
\begin{aligned}
\frac{d}{d t}\left\|\eta_{t}\right\|^{2} \leqq & c\left\|u_{t}\right\|^{2} \cdot\|\widetilde{\Delta} \eta\|^{2}+c\|\widetilde{\Delta} u\|^{2} \cdot\left\|\eta_{t}\right\|^{2}+c\|\nabla \eta\|^{4} \cdot\left\|\eta_{t}\right\|^{2} \\
& +c \lambda_{n+1}^{-1}\left(\left\|u_{t}\right\|^{2}+\left\|\nabla u_{t}\right\|^{2}\right)\|\widetilde{\Delta} u\|^{2}+c \lambda_{n+1}^{-1}\|\widetilde{\Delta} u\|^{2} \cdot\left\|\nabla u_{t}\right\|^{2} .
\end{aligned}
$$

Multiplying by $e^{t}$ and integrating, using (13), and (15), we get

$$
\left\|\eta_{t}(t)\right\|^{2} \leqq e^{-t}\left\|\eta_{t}(0)\right\|^{2}+e^{-t} \int_{0}^{t} e^{\tau} c\left(\gamma^{2}+\lambda_{n+1}^{-1}\right) d \tau+c \lambda_{n+1}^{-1} e^{-t} \int_{0}^{t} e^{\tau}\left\|\nabla u_{t}\right\|^{2} d \tau .
$$

From (14), it is clear $\left\|\eta_{t}(0)\right\|^{2} \leqq c \lambda_{n+1}^{-1}\|\widetilde{\Delta} a\|^{4}$. Using this and the $a$ priori estimates of Lemma 6 , we get

$$
\left\|\eta_{t}(t)\right\|^{2}<c\left(\gamma^{2}+\lambda_{n+1}^{-1}\right) \text {. }
$$

Since

$$
-\frac{d}{d t}\|\nabla \eta\|^{2}=-2\left(\nabla \eta, \nabla \eta_{t}\right)=2\left(\widetilde{\Delta} \eta, \eta_{t}\right) \leqq 1 / 2\|\widetilde{\Delta} \eta\|^{2}+2\left\|\eta_{t}\right\|^{2},
$$

(12) implies

$$
\|\tilde{\Delta} \eta\|^{2} \leqq\left\|\eta_{t}\right\|^{2}+c\|\tilde{\Delta} u\|^{2} \cdot\|\nabla \eta\|^{2}+c\|\nabla \eta\|^{6}+c \lambda_{n+1}^{-1}\|\tilde{\Delta} u\|^{4} .
$$

Clearly (18), (19) and the assumption $\|\nabla \eta\|<\gamma \leqq \delta$ imply

$$
\|\tilde{\Delta} \eta(t)\|^{2}<c\left(\gamma^{2}+\lambda_{n+1}^{-1}\right) \text {. }
$$

6. A-priori estimate of $\left\|g^{n}\right\|$. The forcing term on the right side of (7) is

$$
\begin{aligned}
g^{n}= & Q_{n}\left[u \cdot \nabla \eta^{n}\right]+Q_{n}\left[\eta^{n} \cdot \nabla u\right]+Q_{n}\left[\eta^{n} \cdot \nabla \eta^{n}\right] \\
& +P_{n}\left[\eta^{n} \cdot \nabla e^{n}\right]+P_{n}\left[e^{n} \cdot \nabla \eta^{n}\right]+P_{n}\left[u \cdot \nabla e^{n}\right]+P_{n}\left[e^{n} \cdot \nabla v^{n}\right] .
\end{aligned}
$$


LEMma 9. Whenever $\|\tilde{\Delta \eta}(t)\|^{2}<R\left(\delta^{2}+\lambda_{1}\right)$, there holds

$$
\left\|g^{n}(t)\right\|^{2} \leqq \theta(n)\left\|\widetilde{\Delta} \eta^{n}\right\|^{2}+\nu \lambda_{n+1}^{-1},
$$

with $\theta(n)$ a function satisfying $\theta(n) \rightarrow 0$ as $n \rightarrow \infty$. The function $\theta$ and constant $\nu$ depend only on $\delta, R, \Omega$ and $\sup _{t \geq 0}\|\widetilde{\Delta} u\|$.

To prove Lemma 9, we need:

Lemma 10. For any vector field $w \in H^{1}(\Omega)$, there holds

$$
\left\|Q_{n} w\right\|^{2} \leqq \hat{\theta}(n)\|w\|_{H^{1}}^{2},
$$

with $\hat{\theta}(n)$ a function satisfying $\hat{\theta}(n) \rightarrow 0$ as $n \rightarrow \infty$. The function $\hat{\theta}(n)$ depends only on $\Omega$.

To prove Lemma 10, we need:

LeMma 11. For every $0<\varepsilon<1$, there exists a continuously differentiable, piecewise twice differentiable "cut-off" function $\lambda_{s}(s)$, defined for $s \geqq 0$, such that $\lambda_{s}(0)=1, \lambda_{\varepsilon}^{\prime}(0)=0, \lambda_{\varepsilon}(s)=0$ for $s \geqq \varepsilon$, and such that everywhere $\left|\lambda_{\varepsilon}(s)\right| \leqq \min \{\varepsilon / s, 1\},\left|\lambda_{\varepsilon}^{\prime}(s)\right| \leqq \min \left\{\varepsilon / s, C_{\varepsilon}\right\}$, and $\left|\lambda_{\varepsilon}^{\prime \prime}(s)\right| \leqq C_{\varepsilon}$, with constants $C_{\varepsilon}$ dependent only on $\varepsilon$.

The construction of $\lambda_{\varepsilon}(s)$ is well-known; see [2]. We only remark, it is probably easiest to start with the function

$$
\tilde{\lambda}_{\varepsilon}(s) \equiv \int_{s}^{\varepsilon} \frac{\varepsilon}{\sigma}\left(1-\frac{\sigma}{\varepsilon}\right) d \sigma=\varepsilon(\log \varepsilon-\log s)+(s-\varepsilon),
$$

defined for $0<s \leqq \varepsilon$. Observing that $\left|\tilde{\lambda}_{\varepsilon}(s)\right| \leqq \varepsilon / s,\left|\tilde{\lambda}_{\varepsilon}^{\prime}(s)\right| \leqq \varepsilon / s$, and $\tilde{\lambda}_{:}^{\prime \prime}(s)=\varepsilon / s^{2}$, it is clear how to proceed to construct $\lambda_{s}(s)$.

Proof of Lemma 10. Let $\lambda_{\varepsilon}(x) \equiv \lambda_{\varepsilon}(s)$, where $\lambda_{\varepsilon}(s)$ is as in Lemma 11 and $s=$ distance $(x, \partial \Omega)$. For sufficiently small $\varepsilon$, this is well-defined and the estimates for the derivatives of $\lambda_{\varepsilon}(s)$ are valid for $\lambda_{\varepsilon}(x)$, i.e., $\left|\nabla \lambda_{\varepsilon}(x)\right|=\left|\lambda_{s}^{\prime}(s)\right|$ and $\left|D_{x}^{2} \lambda_{\varepsilon}(x)\right| \leqq c\left(\left|\lambda_{\varepsilon}^{\prime \prime}(s)\right|+\left|\lambda_{s}^{\prime}(s)\right|\right)$.

Choose a vector field $\psi$, in accordance with Lemmas 1 and 2, such that $\operatorname{curl} \psi=P w,\left.\psi\right|_{\partial \Omega}=0$, and $\|\psi\|_{H^{2}} \leqq c\|P w\|_{I^{1}} \leqq c\|w\|_{I I^{1}}$. Clearly,

$$
P w-\operatorname{curl}\left(\lambda_{\varepsilon} \psi\right) \in J_{1}(\Omega), \quad \operatorname{curl}\left(\lambda_{\varepsilon} \psi\right) \in L^{2}(\Omega)
$$

We have the following estimates:

$$
\begin{aligned}
& \left\|P w-\operatorname{curl}\left(\lambda_{\varepsilon} \psi\right)\right\|_{H^{1}}=\left\|P w-\lambda_{\varepsilon} P w-\left(\nabla \lambda_{\varepsilon}\right) \times \psi\right\|_{H^{1}} \\
& \quad \leqq C_{\varepsilon}\|P w\|_{H^{1}}+\left\|\left(\nabla \lambda_{\varepsilon}\right) \times \psi\right\|+\left\|\left(D_{x}^{2} \lambda_{\varepsilon}\right) \psi\right\|+\left\|\left(\nabla \lambda_{\varepsilon}\right) \nabla \psi\right\| \\
& \quad \leqq C_{\varepsilon}\|P w\|_{H^{1}} \leqq C_{\varepsilon}\|w\|_{H^{1}},
\end{aligned}
$$




$$
\begin{aligned}
& \left\|\operatorname{curl}\left(\lambda_{\varepsilon} \psi\right)\right\| \leqq\left\|\nabla \lambda_{\varepsilon} \times \psi\right\|+\left\|\lambda_{\varepsilon} \operatorname{curl} \psi\right\| \\
& \quad \leqq\left(\int_{\Omega_{\varepsilon}}|\varepsilon / s|^{2} \cdot|\psi|^{2} d x\right)^{1 / 2}+\left(\int_{\Omega_{\varepsilon}}|\nabla \psi|^{2} d x\right)^{1 / 2} \\
& \quad \leqq c \varepsilon\|\psi\|_{I I^{1}}+c \varepsilon\|\psi\|_{H^{2}} \leqq c \varepsilon\|w\|_{I I^{1}} .
\end{aligned}
$$

In (25) we have set $\Omega_{\varepsilon} \equiv\{x \in \Omega$ : $\operatorname{dist}(x, \partial \Omega)<\varepsilon\}$ and used the inequality

$$
\int_{0}^{1} \phi^{2}(s) / s^{2} d s \leqq 4 \int_{0}^{1}\left(\phi^{\prime}(s)\right)^{2} d s,
$$

valid if $\dot{\phi}(0)=0$, and also the inequality $\|\dot{\phi}\|_{L^{2}\left(\Omega_{\varepsilon}\right)} \leqq c \varepsilon\|\phi\|_{I^{1}(\Omega)}$, valid for $\phi \in H^{1}(\Omega)$. Finally, using Lemma 4 and (23), (24), (25), we have

$$
\begin{aligned}
\left\|Q_{n} w\right\| & =\left\|Q_{n} P_{w}\right\| \\
& \leqq\left\|Q_{n}\left[P w-\operatorname{curl}\left(\lambda_{\varepsilon} \psi\right)\right]\right\|+\left\|Q_{n} \operatorname{curl}\left(\lambda_{\varepsilon} \psi\right)\right\| \\
& \leqq \lambda_{n+1}^{-1 / 2}\left\|P w-\operatorname{curl}\left(\lambda_{\varepsilon} \psi\right)\right\|+\left\|\operatorname{curl}\left(\lambda_{\varepsilon} \psi\right)\right\| \\
& \leqq \lambda_{n+1}^{-1 / 2} C_{\varepsilon}\|w\|_{I^{1}}+c \varepsilon\|w\|_{I^{1}},
\end{aligned}
$$

which clearly implies the result.

Proof of Lemma 9. Using Lemmas 4,5 and 10 we dotain:

$$
\begin{aligned}
\left\|Q_{n}\left[u \cdot \nabla \eta^{n}\right]\right\|^{2} & \leqq \hat{\theta}(n)\left\|u \cdot \nabla \eta^{n}\right\|_{I^{1}}^{2} \\
& \leqq \hat{\theta}(n)\left(\left\|u \eta_{x}^{n}\right\|^{2}+\left\|u_{x} \eta_{x}^{n}\right\|^{2}+\left\|u \eta_{x x}^{n}\right\|^{2}\right) \\
& \leqq \hat{\theta}(n)\left(\left\|u \eta_{x}^{n}\right\|^{2}+\|\nabla u\|_{4}^{2}\left\|\nabla \eta^{n}\right\|_{4}^{2}+\left\|u \eta_{x x}^{n}\right\|^{2}\right) \\
& \leqq c \hat{\theta}(n)\|\widetilde{\Delta} u\|^{2} \cdot\left\|\widetilde{\Delta} \eta^{n}\right\|^{2},
\end{aligned}
$$$$
\left\|Q_{n}\left[\eta^{n} \cdot \nabla u\right]\right\|^{2} \leqq \hat{\theta}(n)\left\|\eta^{n} \cdot \nabla u\right\|_{H^{1}}^{2}
$$$$
\leqq \hat{\theta}(n)\left(\left\|\eta^{n} u_{x}\right\|^{2}+\left\|\eta_{x}^{n} u_{x}\right\|^{2}+\left\|\eta^{n} u_{x x}\right\|^{2}\right)
$$$$
\leqq c \hat{\theta}(n)\|\widetilde{\Delta} u\|^{2} \cdot\left\|\widetilde{\Delta} \eta^{n}\right\|^{2} \text {, }
$$

$\left\|Q_{n}\left[\eta^{n} \cdot \nabla \eta^{n}\right]\right\|^{2} \leqq \hat{\theta}(n)\left\|\eta^{n} \cdot \nabla \eta^{n}\right\|_{H^{1}}^{2}$

$$
\begin{aligned}
& \leqq \hat{\theta}(n)\left(\left\|\eta^{n} \eta_{x}^{n}\right\|^{2}+\left\|\eta_{x}^{n} \eta_{x}^{n}\right\|^{2}+\left\|\eta^{n} \eta_{x x}^{n}\right\|^{2}\right) \\
& \leqq c \hat{\theta}(n)\left\|\widetilde{\Delta} \eta^{n}\right\|^{4}
\end{aligned}
$$

$\left\|P_{n}\left[\eta^{n} \cdot \nabla e^{n}\right]\right\|^{2} \leqq\left\|\eta^{n} \cdot \nabla e^{n}\right\|^{2} \leqq c\left\|\widetilde{\Delta} \eta^{n}\right\|^{2} \cdot\left\|\nabla e^{n}\right\|^{2} \leqq c \lambda_{n+1}^{-1}\|\widetilde{\Delta} u\|^{2} \cdot\left\|\widetilde{\Delta} \eta^{n}\right\|^{2}$, $\left\|P_{n}\left[e^{n} \cdot \nabla \eta^{n}\right]\right\|^{2} \leqq\left\|e^{n} \cdot \nabla \eta^{n}\right\|^{2} \leqq c\left\|e^{n}\right\|_{6}^{2} \cdot\left\|\nabla \eta^{n}\right\|_{3}^{2} \leqq c \lambda_{n+1}^{-1}\|\widetilde{\Delta} u\|^{2} \cdot\left\|\widetilde{\Delta} \eta^{n}\right\|^{2}$, $\left\|P_{n}\left[u \cdot \nabla e^{n}\right]\right\|^{2} \leqq\left\|u \cdot \nabla e^{n}\right\|^{2} \leqq c\|\Delta u\|^{2} \cdot\left\|\nabla e^{n}\right\|^{2} \leqq c \lambda_{n+1}^{-1}\|\widetilde{\Delta} u\|^{4}$, $\left\|P_{n}\left[e^{n} \cdot \nabla v^{n}\right]\right\|^{2} \leqq\left\|e^{n} \cdot \nabla v^{n}\right\|^{2} \leqq c\left\|e^{n}\right\|_{6} \cdot\left\|\nabla v^{n}\right\|^{2} \leqq c \lambda_{n+1}^{-1}\|\widetilde{\Delta} u\|^{4}$.

7. Proof of Main Result. An $a$-priori estimate, similar to Lemma 6 , will be needed for solutions $\zeta$ of (2). If $\left\|\nabla \zeta\left(t_{0}\right)\right\|<\delta$, (A3) ensures that $\|\nabla \zeta(t)\|<A \delta$, for all $t \geqq t_{0}$. So $v=u+\zeta$ is a solution of the Navier-Stokes equations with $\|\nabla v(t)\|<M+A \delta$, for $t \geqq t_{0}$. 
Also, in view of Lemma $6,\left\|\widetilde{\Delta} v\left(t_{0}\right)\right\| \leqq\left\|\widetilde{\Delta} u\left(t_{0}\right)\right\|+\left\|\widetilde{\Delta} \zeta\left(t_{0}\right)\right\|$ is bounded if $\left\|\widetilde{\Delta} \zeta\left(t_{0}\right)\right\|$ is bounded. Hence the $a$-priori estimates for solutions of the Navier-Stokes equations, already appealed to in Lemma 6, imply $\|\widetilde{\Delta} \zeta(t)\|$ is bounded, for all $t \geqq t_{0}$, in terms of $\left\|\widetilde{\Delta} \zeta\left(t_{0}\right)\right\|$ and the constants and norms of assumptions (A1), (A2), (A3). In summary:

Lemma 12. For perturbations $\zeta$ (i.e., solutions of (2)) which satisfy initially $\left\|\nabla \zeta\left(t_{0}\right)\right\|<\delta$ and $\left\|\widetilde{\Delta} \zeta\left(t_{0}\right)\right\|^{2} \leqq R\left(\delta^{2}+\lambda_{1}^{-1}\right)$, there holds $\|\widetilde{\Delta} \zeta(t)\|<c$, for all $t \geqq t_{0}$, where $c$ is a constant dependent only on $R, \delta, \Omega$ and the bounds assumed for various norms in conditions (A1), (A2), (A3).

Now consider the expression $\beta(t)=c\left(\|\widetilde{\Delta} u(t)\|^{2}+\left\|\widetilde{\Delta} \eta^{n}\right\|^{2}+\|\widetilde{\Delta} \zeta(t)\|^{2}\right)$ appearing in Lemma 7 . In view of Lemmas 6,8 and 12, there exists a constant $B$ bounding all possible values of $\beta(t)$ in any interval $t_{0} \leqq t \leqq t^{*}$ for which it is known

$$
\left\|\nabla \eta^{n}(t)\right\|<\delta,
$$

provided we restrict our considerations to perturbations $\zeta$ satisfying

$$
\left\|\nabla \zeta\left(t_{0}\right)\right\|<\delta \text { and }\left\|\widetilde{\Delta} \zeta\left(t_{0}\right)\right\|^{2}<R\left(\delta^{2}+\lambda_{1}^{-1}\right) .
$$

Consider, also, the forcing term $g^{n}$ appearing in Lemma 7 . Together, Lemmas 8 and 9 imply

$$
\left\|g^{n}(t)\right\|^{2} \leqq \theta(n) R\left(\gamma^{2}+\lambda_{n+1}^{-1}\right)+\nu \lambda_{n+1}^{-1}
$$

on any interval $0 \leqq t \leqq t^{*}$ for which it is known

$$
\left\|\nabla \eta^{n}(t)\right\|<\gamma, \quad \text { with } \gamma \leqq \delta .
$$

We now chosse $T$ sufficiently large that

$$
A e^{-\alpha T} \leqq \frac{1}{2},
$$

with $\alpha$ and $A$ as in (A3), and then choose $N$ sufficiently large that

$$
e^{B T} T \theta(n) R<\frac{1}{8}
$$

and

$$
\gamma_{n} \equiv \sqrt{8}\left(e^{B T} T \theta(n) R+\nu\right)^{1 / 2} \lambda_{n+1}^{-12}<\delta
$$

hold for $n \geqq N$. Here, we have defined $\gamma_{n}$ in such a way that, in the presence of (31), there holds

$$
e^{B T} T\left[\theta(n) R\left(\gamma_{n}^{2}+\lambda_{n+1}^{-1}\right)+\nu \lambda_{n+1}^{-1}\right]<\frac{1}{4} \gamma_{n}^{2},
$$


as may be easily checked.

For $n \geqq N$, we claim

$$
\left\|\nabla \eta^{n}(t)\right\|<\gamma_{n}, \quad \text { for all } t \geqq 0 \text {. }
$$

If not, that is, if (34) fails for some $n \geqq N$, let $t^{*}$ be the first value of $t$ for which $\left\|\nabla \eta^{n}\left(t^{*}\right)\right\|=\gamma_{n}$. To show it is impossible that $t^{*} \leqq T$, consider Lemma 7 with $t_{0}=0$ and $\zeta \equiv 0$. The implication, in view of (28) and (33), and remembering $\eta^{n}(0)=0$, is that

$$
\begin{aligned}
\left\|\nabla \eta^{n}\left(t^{*}\right)\right\|^{2} & =\left\|\nabla w\left(t^{*}\right)\right\|^{2} \\
& \leqq e^{E T} T\left[\theta(n) R\left(\gamma_{n}^{2}+\lambda_{n+1}^{-1}\right)+\nu \lambda_{n \cdot+1}^{-1}\right]<\frac{1}{4} \gamma_{n}^{2},
\end{aligned}
$$

which contradicts our supposition about $t^{*}$. On the other hand, if $t^{*}>T$, then $\left\|\nabla \eta^{n}\left(t^{*}-T\right)\right\|<\gamma_{n}$, and of course $\left\|\tilde{\Delta \eta} \eta^{n}\left(t^{*}-T\right)\right\|<$ $R\left(\delta^{2}+\lambda_{\perp}^{-1}\right)$, by Lemma 8. So, considering Lemma 7 again, but with $t_{0}=t^{*}-T$ and $\zeta\left(t^{*}-T\right)=\eta^{n}\left(t^{*}-T\right)$, we find

$$
\begin{aligned}
\left\|\nabla \eta^{n}\left(t^{*}\right)-\zeta\left(t^{*}\right)\right\|^{2} & \leqq e^{B T} T\left[\theta(n) R\left(\gamma_{n}^{2}+\lambda_{n+1}^{-1}\right)+\nu \lambda_{n}^{-1}\right] \\
& \leqq \frac{1}{4} \gamma_{n}^{2} .
\end{aligned}
$$

In addition, in view of (30), the assumption (A3) implies

$$
\left\|\nabla \zeta\left(t^{*}\right)\right\| \leqq \frac{1}{2}\left\|\nabla \zeta\left(t^{*}-T\right)\right\|<\frac{1}{2} \gamma_{n} .
$$

Together, (35) and (36) imply $\left\|\nabla \eta^{n}\left(t^{*}\right)\right\|<\gamma_{n}$, again contradicting our supposition about $t^{*}$. So (34) must hold, and combined with Lemma 6 , it implies the theorem of $\S 3$.

\section{REFERENCES}

1. L. Cattabriga, Su un prob̉leme al contorno relativo al sistema di equazioni di Stokes, Rend. Sem. Mat. Univ. Padova, 31 (1961), 308-340.

2. R. Finn, On the steady-state solutions of the Navier-Stokes equations, Acta Math., 3 (1961), 197-244.

3. C. Foias, Statistical study of Navier-Siokes equations, Part I, Rend. Sem. Math. Univ. Padova, 48 (1973), 219-348.

4. J. Heywood, The Navier-Stokes equations: On the existence, regularity and decay of solutions, Indiana U. Math. J., 29 (1980), 639-681.

5. - Classical solutions of the Navier-Stokes equations, Proc. IUTAM Symp, Paderborn (W. Germany) 1979, Springer Lecture Notes in Math., 771 (1980).

6. - On classical solutions of the nonstationary Navier-Stokes equations in two and three dimensions, Fluid Dynamics Transactions, 10, (1980), 177-203.

7. J. Heywood and R. Rannacher, Finite element approximation of the nonstationary Navier-Stokes Problem I, SIAM J. Num. Anal.

8. E. Hopf, Über die Anfangswertaufgabe für die hydrodynamischen Grundgleichungen, Math. Nachr., 4 (1951), 213-231. 
9. S. Ito, The existence and the uniqueness of regular solution of nonstationary NavierStokes equation, J. Fac. Sci. Univ. Tokyo Sect. IA, 9 (1961), 103-140.

10. A. A. Kiselev and O. A. Ladyzhenskaya, On the existence and uniqueness of the solution of the nonstationary problem for a viscous incompressible fluid, Izv. Akad. Nauk SSSR Ser. Mat., 21 (1957), 665-680.

11. O. A. Ladyzhenskaya, The mathematical theory of viscous incompressible flow, Second Edition, Gordon and Breach, New York, 1969.

12. J. L. Lions, Quelques méthodes de résolution des problèmes aux limites nonlineaires, Dunod (1969), Paris.

13. J. Necas, Les méthodes directes en thóorie des équations elliptiques, Masson et $C^{10}$, Editeurs, (1967), Paris.

14. G. Prodi, Teoremi di tipo locale per il sis'ema de Navier-Stokes e stabilità delle soluzione stazionarie, Rend. Sem. Mat. Univ. Padova, 32 (1962), 374-397.

15. R. Rautmann, On the convergence-rate of nonstationary Navier-Stokes approximations, Proc. IUTAM Symp, Paderborn (W. Germany) 1979, Springer Lecture Notes in Math., 771 (1980).

16. - Eine Fehlerschranke für Galerkinapproximationen lokaler Navier-StokesLösungen, Constructive methods for Nonlinear Boundary Value Problems and Nonlinear Oscillations, edited by J. Albrecht, L. Collatz, K. Kirchgässner, Basel IRNN 48, Birkhauser, (1979), 110-125.

17. R. Temam, Navier-Stokes Equations, North-Holland Publ. Comp., Amsterdam, 1977.

Received August 20,1980. This research was supported by Natural Sciences and Engineering Research Council Canada, grant no. A4150

UNIVERSITY OF BRITISH COLUMEIA

VANCOUVER, BC, CANADA V6T1W5 



\title{
PACIFIC JOURNAL OF MATHEMATICS
}

\section{EDITORS}

\author{
DONALD BABBITT (Managing Editor) \\ University of California \\ Los Angeles, CA 90024 \\ Hugo RossI \\ University of Utah \\ Salt Lake City, UT 84112 \\ C. C. MOORE and ANDREW OGG \\ University of California \\ Berkeley, CA 94720
}

J. DugundjI

Department of Mathematics

University of Southern California

Los Angeles, CA 90007

R. FinN and J. Milgram

Stanford University

Stanford, CA 94305

\section{ASSOCIATE EDITORS}
R. ARENS
E. F. Beckenbach
B. H. NeumanN
F. WOLF
K. YoSHIDA

\section{SUPPORTING INSTITUTIONS}

\author{
UNIVERSITY OF ARIZONA \\ UNIVERSITY OF BRITISH COLUMBIA \\ CALIFORNIA INSTITUTE OF TECHNOLOGY \\ UNIVERSITY OF CALIFORNIA \\ MONTANA STATE UNIVERSITY \\ UNIVERSITY OF NEVADA, RENO \\ NEW MEXICO STATE UNIVERSITY \\ OREGON STATE UNIVERSITY
}

\author{
UNIVERSITY OF OREGON \\ UNIVERSITY OF SOUTHERN CALIFORNIA \\ STANFORD UNIVERSITY \\ UNIVERSITY OF HAWAII \\ UNIVERSITY OF TOKYO \\ UNIVERSITY OF UTAH \\ WASHINGTON STATE UNIVERSITY \\ UNIVERSITY OF WASHINGTON
}

The Supporting Institutions listed above contribute to the cost of publication of this Journal, but they are not owners or publishers and have no responsibility for its content or policies.

Mathematical papers intended for publication in the Pacific Journal of Mathematics should be in typed form or offset-reproduced, (not dittoed), double spaced with large margins. Please do not use built up fractions in the text of the manuscript. However, you may use them in the displayed equations. Underline Greek letters in red, German in green, and script in blue. The first paragraph or two must be capable of being used separately as a synopsis of the entire paper. Please propose a heading for the odd numbered pages of less than 35 characters. Manuscripts, in triplicate, may be sent to any one of the editors. Please classify according to the scheme of Math. Reviews, Index to Vol. 39. Supply name and address of author to whom proofs should be sent. All other communications should be addressed to the managing editor, or Elaine Barth, University of California, Los Angeles, California, 90024.

50 reprints to each author are provided free for each article, only if page charges have been substantially paid. Additional copies may be obtained at cost in multiples of 50 .

The Pacific Journal of Mathematics is issued monthly as of January 1966. Regular subscription rate: $\$ 102.00$ a year (6 Vols., 12 issues). Special rate: $\$ 51.00$ a year to individual members of supporting institutions.

Subscriptions, orders for numbers issued in the last three calendar years, and changes of address shoud be sent to Pacific Journal of Mathematics, P.O. Box 969, Carmel Valley, CA 93924, U.S.A. Old back numbers obtainable from Kraus Periodicals Co., Route 100, Millwood, NY 10546.

PUBLISHED BY PACIFIC JOURNAL OF MATHEMATICS, A NON-PROFIT CORPORATION

Printed at Kokusai Bunken Insatsusha (International Academic Printing Co., Ltd.). 8-8, 3-chome, Takadanobaba, Shinjuku-ku, Tokyo 160, Japan. 


\section{Pacific Journal of Mathematics}

\section{Vol. 98, No. 2 \\ April, 1982}

Thomas E. Armstrong, Barycentric simplicial subdivision of infinite-dimensional simplexes and octahedra

Hom Nath Bhattarai and James William Fernandez, Joins of double coset

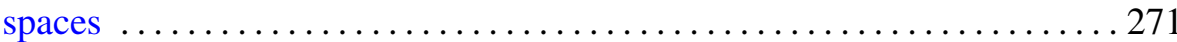

Alexandru Buium, Ritt schemes and torsion theory $\ldots \ldots \ldots \ldots \ldots \ldots \ldots 281$

Jacob Burbea, Operator-valued Pick's conditions and holomorphicity ..... 295

Su-Shing Chen, Duality condition and property $(\mathrm{S}) \ldots \ldots \ldots \ldots \ldots \ldots . \ldots . \ldots 313$

Ky Fan, Evenly distributed subsets of $S^{n}$ and a combinatorial application . . 323

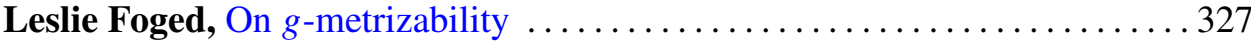

John Groves Heywood, An error estimate uniform in time for spectral

Galerkin approximations of the Navier-Stokes problem ............ 333

Aggie Ho, The Krê̆ n-Milman property and complemented bushes in

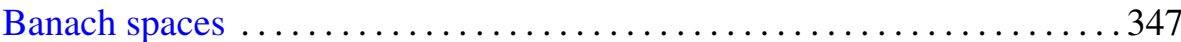

David R. Jackett, Rings on certain mixed abelian groups ............. 365

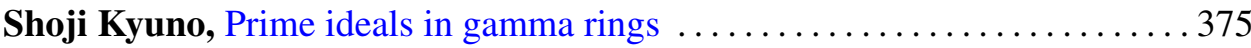

George Lucius O'Brien, Zero-inducing functions on finite abelian

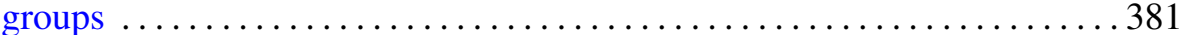

P. Robba, Sur les équations différentielles linéaires $p$-adiques. II ......... 393

Wolfgang Ruess, [Weakly] compact operators and DF spaces ...........419

Claude Schochet, Topological methods for $C^{*}$-algebras. II. Geometry resolutions and the Künneth formula

Harry F. Smith, Jr., Equivalent nilpotencies in certain generalized right

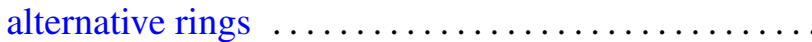

Max Warshauer, Diagonalization up to Witt 469

Hugh C. Williams, A class of primality tests for trinomials which includes the Lucas-Lehmer test 